\title{
Mixed Convection at a Vertical Plate in a Porous Medium with Magnetic Field and Variable Viscosity
}

\author{
N. Veerraju ${ }^{l}$, K.S. Srinivasa Babu ${ }^{2}$ and C.N.B. Rao ${ }^{\dagger}$ \\ ${ }^{1,2}$ Assistant Professor, Dept. of Eng. Mathematics \& Humanities, S.R.K.R. Eng. College, Bhimavaram, \\ 534204, India \\ ${ }^{\dagger}$ Professor, Dept. of Eng. Mathematics \& Humanities, S.R.K.R. Eng. College, Bhimavaram, 534204, India \\ †Corresponding Author Email: cnbrao.bvrm@gmail.com
}

(Received November 11, 2010; accepted January 31, 2011)

\begin{abstract}
A numerical study of a mixed convection boundary layer flow on a vertical plate in a porous medium with magnetic field, variable wall temperature and variable viscosity is made in this paper using the Darcy model. A free stream that varies as a power function of distance along the plate is assumed to flow parallel to the plate. Similarity solutions are obtained for the problem for both assisting flow and opposing flow. In the opposing flow case dual solutions are obtained for certain values of the parameters, and occurrence of boundary layer separation is also observed. Significant differences are observed between the behaviors of the two solutions of the dual solution case. Critical values of the mixed convection parameter are also obtained beyond which there exists no solution for the problem. Some of the observations of the analysis are - the range of values of the mixed convection parameter over which solutions exist for the problem is more in the presence of magnetic field than in its absence and also in the variable wall temperature case than in the isothermal case. Both local drag coefficient and heat transfer coefficient assume only positive values in the isothermal case while they assume both positive and negative values in the varying wall temperature case. Drag is less in the presence of magnetic field than in its absence and also in the isothermal case than in the varying wall temperature case. Heat transfer coefficient diminishes in the absence of magnetic field than in the presence of magnetic field.
\end{abstract}

Keywords: Mixed convection, Variable viscosity, Magnetic field, Varying wall temperature.

\section{NOMENCLATURE}

$B_{0} \quad$ Magnetic flux

C Magnetic interaction parameter, $\frac{K^{*}}{K^{*}+M^{2}}$

$f \quad$ Dimensionless stream function

$g \quad$ Acceleration due to gravity

$h \quad$ Local heat transfer coefficient

$k_{m} \quad$ Effective Thermal conductivity of the saturated porous medium

$K^{*}$ Porous parameter, $\frac{L^{2}}{K}$

K Permeability

$M^{2}$ Hartmann number, $\frac{B_{0}^{2} L^{2} \sigma}{\mu_{\infty}}$

$p \quad$ Pressure

$R a_{x}$ Rayleigh number, $\frac{\rho_{\infty} K g \beta\left(T_{0}-T_{\infty}\right) x}{v_{\infty} \alpha_{m}}$

$T \quad$ Temperature
$T_{e} \quad$ Constant $=T_{\infty}-\frac{1}{\gamma}$

$T_{0} \quad$ Temperature of plate

$T_{\infty} \quad$ Ambient temperature

$u, v \quad$ Velocity components in $x$ - and $y$-directions

$x, y$ Cartesian coordinates

$a \quad$ Constant defined in the equation $\gamma=a \mu_{\infty}$

$U_{\infty} \quad$ Free stream velocity

$P e_{x} \quad \mathrm{Pe}^{\prime}$ clet Number, $\frac{U_{\infty} x}{\alpha_{m}}$

\section{GREEK SYMBOLS}

$\alpha_{m} \quad$ Effective thermal diffusivity of the porous medium

$\beta \quad$ Coefficient of thermal expansion

$\eta \quad$ Similarity variable

$\theta \quad$ Dimensionless temperature

$\theta_{e} \quad$ Viscosity variation parameter 


\author{
$\mu \quad$ Dynamic viscosity \\ $\mu_{\infty} \quad$ Viscosity of the ambient fluid \\ $\rho \quad$ Fluid density \\ $\rho_{\infty} \quad$ Density of the ambient fluid \\ $\psi \quad$ Stream function \\ $\lambda$ Power of index of plate temperature
}

\section{INTRODUCTION}

Extensive studies of mixed convection in porous media have been performed over the last five decades covering a broad range of fields including several different physical effects since such studies have important applications in various fields of science and technology. Mixed convection flows arise when, besides buoyancy, there acts an external force, because of which there will be a free stream or a moving boundary in such problems. In mixed convection studies, there can arise two types of flows - assisting flow and opposing flow, depending on whether the plate is hot or cold as compared to the ambient temperature and based on the direction of the free stream or the moving boundary. Minkowycz et al. (1976) and Cheng et al. (1975) have presented similarity solutions for free convection in a porous medium adjacent to vertical and horizontal plates with wall temperatures being power functions of distance. Using Darcy's law, a boundary layer model for natural convection in a porous medium was studied by Cheng et al. (1977), using Boussinesq's approximation and a similarity transformation. Merkin (1980) is perhaps the first to have discussed assisting and opposing flows in mixed convection at a vertical plate in a porous medium. He has identified the occurrence of dual solutions for certain values of the parameters and also the occurrence of boundary layer separation. Ranganathan et al. (1984) have studied mixed convection boundary layer flow along a vertical surface in a porous medium considering the effects of inertia, porosity variation and blowing at the surface. Joshi et al. (1985) have discussed mixed convection in porous media adjacent to a vertical uniform heat flux surface. Lai et al. (1990) discussed the effect of variable viscosity on convective heat transfer in three different cases of natural convection, mixed convection and forced convection, taking fluid viscosity to vary inversely with temperature. In that work, while discussing mixed convection, the authors have taken only assisting flow into consideration. Chen et al. (1990) have studied the combined effect of quadratic drag, boundary friction, thermal dispersion and non uniform porosity on aiding flow at a vertical surface. Carey et al. (1980) used a regular perturbation analysis to study the effect of variable viscosity on convection in free flow at a vertical plate when the temperature of the plate varies as a power function of distance along the plate. The cases of freely rising plane plume, the flow above a horizontal line source on an adiabatic surface and the flow adjacent to a vertical uniform plane surface were considered and the effect of variable viscosity was discussed. Pu et al. (1999) made an experimental study of mixed convection heat transfer in vertical packed channels. The authors presented possible ranges of values of the mixed convection parameter $R P$ appropriate for free convection, forced $v \quad$ Kinematic viscosity

$\gamma$ Constant defined in equation $\gamma=a \mu_{\infty}$

\section{SUBSCRIPTS}

0 Condition at the plate

$\infty$ Condition at the infinite

convection and mixed convection as $R P>105, R P<1$ and $1<R P<105$ respectively. They also discussed the transition from mixed convection to forced convection or to free convection.

Aly et al. (2003) studied mixed convection boundary layer flow over a vertical surface embedded in a porous medium. Taking the plate temperature to vary as a power function of distance along the plate, similarity solutions of the problem were obtained as functions of two parameters $\lambda$, a parameter that describes variation of the wall temperature and $\varepsilon$, a mixed convection parameter. In the opposing flow case, dual solutions were obtained for certain values of the parameters and also critical values of $\varepsilon$ beyond which no solution exists. Chin et al. (2007) studied the effect of variable viscosity on mixed convection boundary layer flow over a vertical surface embedded in a porous medium. Taking fluid viscosity to vary as an inverse linear function of temperature, similarity solutions were obtained as functions of two parameters $\theta_{e}$, a viscosity variation parameter and $\varepsilon$, mixed convection parameter. The occurrence of boundary layer separation and the existence of dual solutions in the opposing flow case were reported.

In view of possible applications, effect of magnetic field on different convective problems was studied by several researchers. Sobha et al. (2003) studied free convective heat transfer in a porous medium subjected to a magnetic field when the plate temperature varies as a power function of the vertical coordinate. Acharya et al. (2000) studied the effect of magnetic field on free convection in a porous medium with constant heat flux. Rao et al. (2010) studied mixed convection in a porous medium with magnetic field, variable viscosity and varying wall temperature. Taking linear variation of viscosity, the authors have discussed the effect of magnetic field, variable viscosity and varying wall temperature on the mixed convection flow both in the assisting and opposing flow cases.

In the present analysis steady mixed convection boundary layer flow over a vertical impermeable plate embedded in a porous medium is studied. A magnetic field of uniform intensity is assumed to act normal to the plate, temperature of the plate is assumed to vary as a power function of distance along the plate and viscosity of the fluid is assumed to vary inversely as a linear function of temperature. A free stream is assumed to flow parallel to the plate. It is an established fact that similarity for such a mixed convection problem exists only if the free stream also varies as the same power function of distance along the plate as that of the plate temperature. Under this assumption, similarity solutions are obtained for the problem. In the opposing flow case, the existence of dual solutions is also noticed. For a given value of the mixed convection parameter ( $R P)$, 
depending on the values of the other parameters, either a unique solution or dual solution or no solution exist for the problem. Also there exist a critical value of $R P$ beyond which no solution exists. Critical values of $R P$ as well as ranges of values of the parameters for which either a unique solution, dual solution or no solution exist for the problem are determined. Certain qualitatively and quantitatively interesting results are presented in the form of graphs and tables. The behaviors of the solutions including the slip velocity, shear stress, heat transfer coefficient, fluid velocity, temperature, hydrodynamic boundary layer thickness and thermal boundary layer thickness are discussed as functions of parameters of the problem.

\section{FORMULATION}

An isothermal plate immersed vertically in a porous medium saturated with a viscous incompressible homogeneous quiescent fluid is considered. A point ' $O$ ' in the plate is taken as origin of a coordinate system, a vertical line through ' $O$ ' along the plate is taken as $X$ - axis and a line perpendicular to the plate through ' $O$ ' is taken as $Y$ - axis. A magnetic field of uniform intensity $\left(B_{0}\right)$ is applied in a direction normal to the plate. The temperature of the plate $\left(T_{0}\right)$ is assumed to vary as a power function of distance along the plate as

$T_{0}=T_{\infty}+A x^{\lambda}$

where $T_{\infty}$ is the temperature of the ambient fluid, $A$ is a constant, and $\lambda$ is a real number. $\lambda$ is referred to as power of index of wall temperature. The viscosity of the fluid is taken to be an inverse linear function of temperature as

$\frac{1}{\mu}=\frac{1}{\mu_{\infty}}\left\{1+\gamma\left(T-T_{\infty}\right)\right\}$

where $\mu$ is dynamic viscosity, $\mu_{\infty}$ is viscosity evaluated at ambient temperature, $T$ is the temperature of the fluid and $\gamma$ is a constant depending on the viscosity of the fluid defined by

$\gamma=a \mu_{\infty}$

In general, the constant ' $a$ ' takes positive values for liquids and takes negative values for gases. Density of the fluid is assumed to be variable only in the body force term and other fluid properties are assumed to be constant. The ambient fluid is assumed to flow with a velocity $U_{\infty}$ parallel to the plate and this velocity is assumed to vary as a power function of distance $(x)$ along the plate as

$U_{\infty}=b x^{\lambda}$.

Using the Darcy model, the equations governing the steady mixed convection boundary layer flow are well known and are not presented here.

Appropriate boundary conditions on the fluid velocity and fluid temperature are at $Y=0, \quad v=0, \quad T=T_{0}$

at $Y \rightarrow \infty, \quad u \rightarrow U_{\infty}, T \rightarrow T_{\infty}$

Boussinesq's approximation is used, and a stream function $\psi$ is introduced through the relations

$u=\frac{\partial \psi}{\partial y}, v=-\frac{\partial \psi}{\partial x}$

so that, after elimination of pressure, the following equation is obtained

$\left(T-T_{e}\right) \frac{\partial^{2} \psi}{\partial y^{2}}=\frac{\partial \psi}{\partial y} \frac{\partial T}{\partial y}+\left(T-T_{e}\right)^{2} a K \rho_{\infty} g \beta \frac{\partial T}{\partial y}-$

$\sigma B_{0}{ }^{2}\left(T-T_{e}\right)^{2} a K \frac{\partial^{2} \psi}{\partial x^{2}}$

Here

$T_{e}=T_{\infty}-\frac{1}{\gamma}$

Introducing a similarity variable $\eta$ and nondimensional variables $f, \theta$ through the relations

$\eta=\left(\frac{P e_{x}}{2}\right)^{\frac{1}{2}} \frac{y}{x}, \quad \psi=\alpha\left(2 P e_{x}\right)^{\frac{1}{2}} f(\eta), \quad \theta=\frac{T-T_{\infty}}{T_{0}-T_{\infty}}$

where

$P e_{x}=\frac{U_{\infty} x}{\alpha_{m}}$

the governing equations are obtained as

$\frac{f^{\prime \prime}\left(\theta-\theta_{e}\right)-f^{\prime} \theta^{\prime}}{\left(\theta-\theta_{e}\right)^{2}}=-\frac{\theta^{\prime}}{\theta_{e}} R P+\frac{(1-C)}{C} \frac{1}{\theta_{e}} f^{\prime \prime}$

$\theta^{\prime \prime}=-\theta^{\prime} f$

Here a dash denotes derivative with respect to $\eta$,

$R P=\frac{R a_{x}}{P e_{x}}$ is the mixed convection parameter where $R a_{x}=\frac{K g \beta\left(T_{0}-T_{\infty}\right) x}{v_{\infty} \alpha_{m}}$ is a local Rayleigh number,

$P e_{x}$ is local $P e^{\prime}$ clet number and $C=\frac{K^{* *}}{K^{*}+M^{2}}$ is

magnetic interaction parameter, where $M^{2}=\frac{B_{o}{ }^{2} L^{2} \sigma}{\mu_{\infty}}$ is Hartmann number.

The boundary conditions in terms of non-dimensional variables are

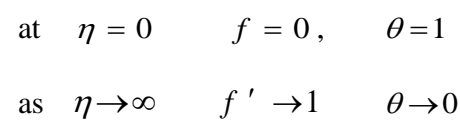

Equation (6) can be integrated once with respect to $\eta$ using the condition on $f^{\prime}$ at infinity to get 


$$
f^{\prime}=\frac{-\left(\theta-\theta_{e}\right)(C \cdot R P \cdot \theta+1)}{\theta_{e}+(C-1) \theta}
$$

Evaluation of this expression at $\eta=0$ gives the slip velocity.

\subsection{Parameters of the Problem}

The parameters of the problem are $R P, C, \lambda$ and $\theta_{e}$. $R P$ the mixed convection parameter takes positive values when the plate is hot i.e., when plate temperature is more than the ambient temperature, and takes negative values when the plate is cold i.e., when plate temperature is less than the ambient temperature. In the present work, by choice, positive values of $R P$ correspond to assisting flow and negative values correspond to opposing flow.

$C$ is the magnetic interaction parameter. In view of its definition, the parameter can take only positive values that are less than or equal to unity. The value unity corresponds to the case when there is no magnetic field and diminishing values of $C$ correspond to increasing intensity of the magnetic field or increasing permeability (i.e., diminishing values of the porous parameter). An increase in the intensity of the magnetic field diminishes the fluid flow and hence is the case with diminishing values of $C$.

The constant ' $A$ ' appearing in the expression for the plate temperature can take positive as well as negative values. $\lambda$ is power of index of wall temperature. This parameter can take positive as well as negative values which give rise to plate temperatures higher or lower than that of ambient temperature depending on the value of ' $A$ '. Zero value of $\lambda$ corresponds to the isothermal case.

The other parameter $\theta_{e}$ is the viscosity variation parameter. It can take positive as well as negative values, of which positive values correspond to gases and negative values correspond to liquids. The value infinity corresponds to constant viscosity case. In this problem, it is observed that 1000 is a large value of $\theta_{e}$ that gives sufficiently accurate constant viscosity results. It is not necessary to differentiate between the values $+\infty$ and $-\infty$ of $\theta_{e}$ and hence between the values +1000 and -1000 .

\subsection{Solution of the Problem}

The Eqs. (7) \& (9) subject to conditions (8) constitutes a boundary value problem. This problem is solved by Runge-Kutta- Gill method together with a shooting technique and solutions are obtained only for negative values of $R P$, since its negative values correspond to opposing flow and it is intended to confine attention to the opposing flow case. It may be further noted that the value 1000 for $\theta_{e}$ has given results that coincide with those corresponding to the constant viscosity case. Solutions in certain appropriate cases are compared with available results of the literature. Important quantities of physical interest such as slip velocity, fluid velocity, fluid temperature, shear stress, heat transfer coefficient, hydrodynamic boundary layer thickness and thermal boundary layer thickness are determined. Quantitatively and qualitatively interesting behaviors of the solutions are presented through figures and tables and discussed as functions of the parameters.

\section{DISCUSSION OF THE RESULTS}

In this paper, attention is paid to the opposing flow case and hence on the results for negative values of mixed convection parameter $R P$. Certain important results are presented in the form of Figs. 1 to 7 and some more results in the form of Tables 1 to 2 .

In what follows the shear stress at the plate, ' $f$ " $(0)$ ' is referred to as skin friction and the heat transfer rate at the plate, ' $-\theta^{\prime}(0)$ ' is referred to as the wall heat transfer rate.

Local drag coefficient is defined as

$C d_{x}=\frac{\left.\mu \frac{\partial u}{\partial y}\right|_{y=0}}{\rho_{\infty}\left(U_{\infty}\right)^{2}}=\frac{v_{\infty}}{\left(1+\gamma A x^{\lambda}\right)} \frac{\left(P e_{x}\right)^{\frac{-1}{2}}}{\alpha \sqrt{2}} f^{\prime \prime}(0)$

Local Nusselt number is defined as

$N u_{x}=\frac{-K\left(\frac{\partial T}{\partial y}\right)_{y=0}}{T_{0}-T_{\infty}}=\frac{-K}{x}\left(\frac{P e_{x}}{2}\right)^{\frac{1}{2}} \theta^{\prime}(0)$

The discussion of the results is presented in two parts (i) Isothermal $(\lambda=0)$ i.e., constant wall temperature case and (ii) Non- Isothermal $(\lambda \neq 0)$ i.e., varying wall temperature case.

\subsection{Skin Friction \& Heat Transfer Coefficient (Isothermal Case, $\lambda=0$ )}

Variations in ' $f$ " $(0)$ ' with the mixed convection parameter are presented in Figs. 1(a), 1(b) and the corresponding variations in ' $-\theta^{\prime}(0)$ ' are presented in Figs 2(a), 2(b). It may be noted that, skin friction ' $f^{\prime \prime}(0)$ ' takes increasing values with increasing negative values of $R P$ up to a certain stage and diminish beyond that stage. It is also noted that there is a critical value of $R P$ beyond which there exist no solution. From the plots of ' $f$ " $(0)$ ', it may noted that there can be a unique solution, dual solutions or no solution for the problem depending on the values of $R P$. In the case of dual solutions, the solution corresponding to a relatively larger value of " $f$ " $(0)$ ' is referred to as the upper solution and the one corresponding to a smaller value of ' $f$ " $(0)$ ' is referred to as the lower solution.

Optimum values for ' $f$ " $(0)$ ' occurs when $\theta_{e}=-2$ and this optimum diminishes as $\theta_{e}$ changes from -2 to -8 and also from 1000 to 2 . The range values of $R P$ over which solution exists for the problem is maximum for $\theta_{e}=2$, and the range diminishes as $\theta_{e}$ increases from 2 to 1000 . The range further diminishes as $\theta_{e}$ changes from -8 to -2 . 
Both in the presence ( $C=0.5$ ) as well as in the absence of magnetic field ( $C=1$ ), behavior of " $f$ " (0) ' is the same, except that the range of values of $R P$ over which solution exists is considerably larger in the presence of magnetic field.

From Figs. 2(a), 2(b) it may be noted that, unlike ' $f^{\prime \prime}(0)$ ', ' $-\theta^{\prime}(0)$ ' takes diminishing values with increasing negative values of $R P$ up to the critical value and diminishes further as $R P$ increases. Since zero value of $R P$ corresponds to forced convection, we may note that the heat transfer coefficient of the mixed convection case assumes smaller numerical values than the one in the forced convection case. Unlike ' $f$ " $(0)$ ', ' $-\theta^{\prime}(0)$ 'assumes smaller values as $\theta_{e}$ changes from 2 to 1000 or from -2 to -8 . Effect of magnetic field on the heat transfer coefficient is not as significant as on skin friction.

\subsection{Skin Friction \& Heat Transfer Coefficient (Varying Wall Temperature Case, $\lambda \neq 0$ )}

Variations in ' $f$ " $(0)$ ' of this case are presented in Figs. 1(c), 1(d) and corresponding variations in " $-\theta^{\prime}(0)$ ' are presented in Figs. 2(c), 2(d).

In the isothermal case ' $f$ " $(0)$ ' as well as ' $-\theta^{\prime}(0)$, are observed to assume only positive values, while in the present varying wall temperature case $(\lambda=0.05)$, they can be observed to assume both positive and negative values indicating that dual solutions exist over a wider range of values of $R P$ in the varying wall temperature case than in isothermal case. Variations in skin friction and heat transfer coefficient of the present case with the mixed convection parameter $R P$ and viscosity variation parameter $\theta_{e}$ are similar to the corresponding variations of the isothermal case.

The range of values of $R P$ over which solution exists for the problem in this case $(\lambda=0.05)$ is slightly more than the corresponding range in the isothermal case $(\lambda=0.0)$ for a given set of values of the other parameters. In the presence of magnetic field $(C=0.5)$ the range over which solutions exists is considerably larger than the range in the absence of magnetic field $(C=1)$.

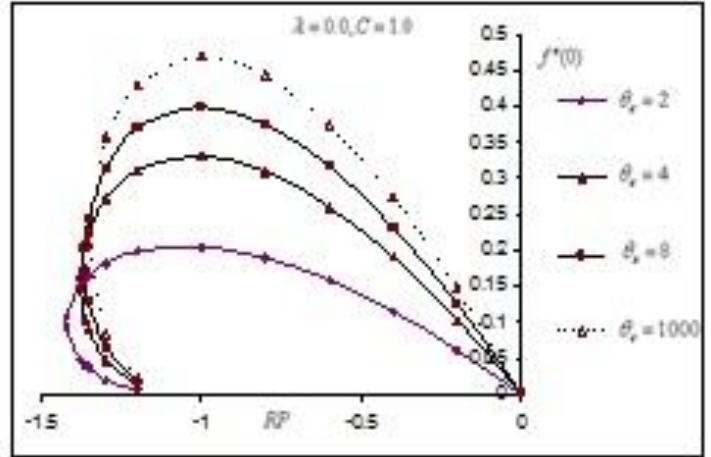

(a)

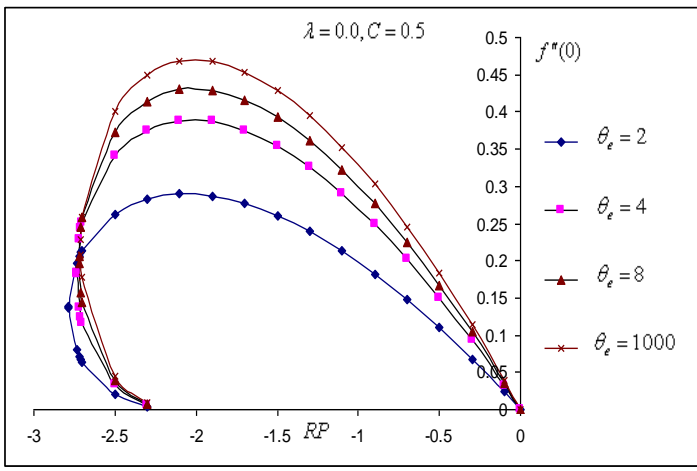

(b)

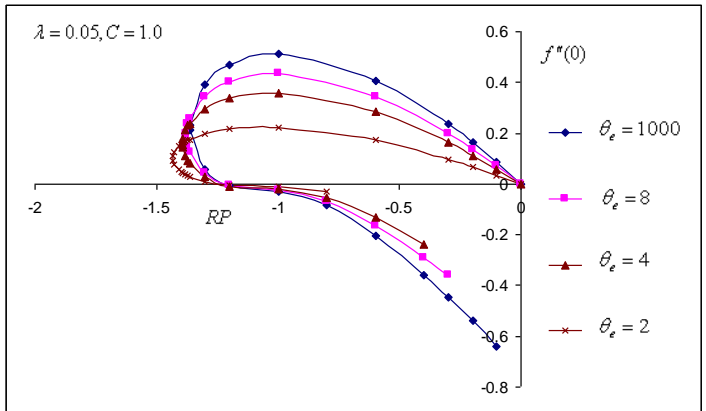

(c)

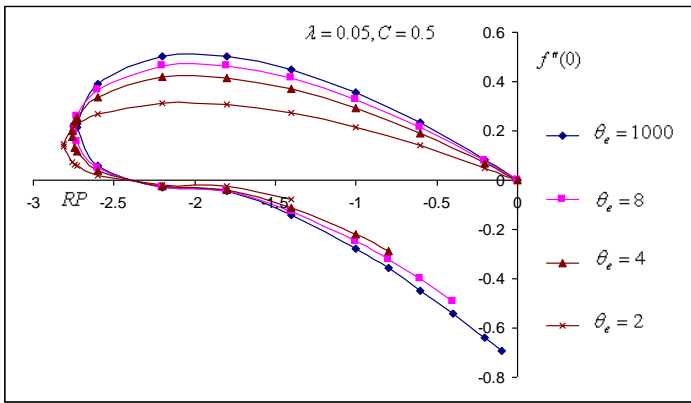

(d)

Fig.1. Variations in $f^{\prime \prime \prime}(0)$ for positive values of $\theta_{\mathrm{e}}$. (a) $\lambda=0.0, \mathrm{C}=1.0$; (b) $\lambda=0.0, \mathrm{C}=0.5$; (c) $\lambda=0.05, \mathrm{C}=1.0$; (d) $\lambda=0.05, \mathrm{C}=0.5$ 


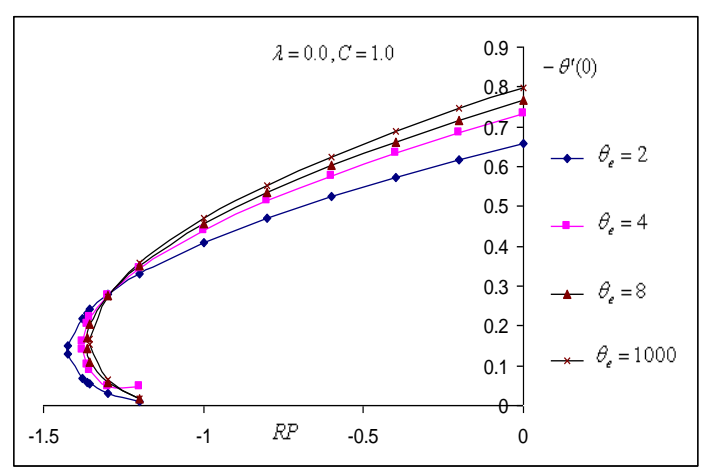

(a)

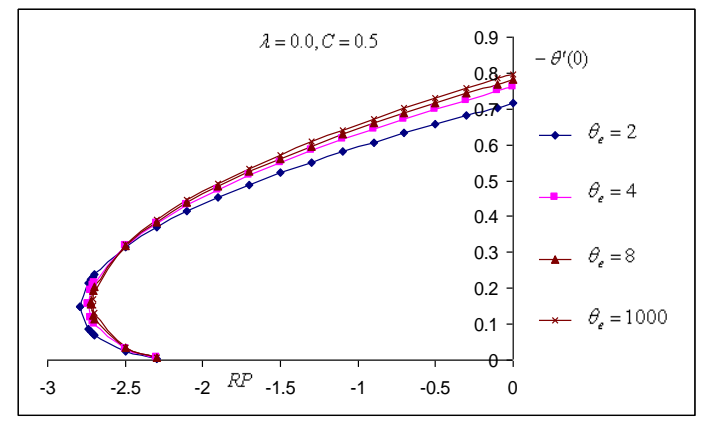

(b)

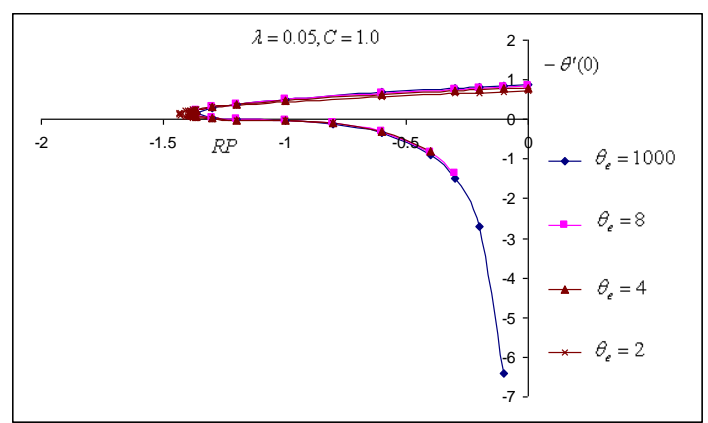

(c)

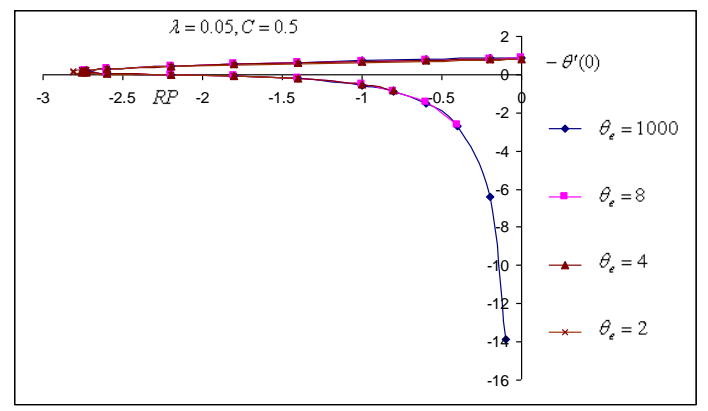

(d)

Fig. 2. Variations in ' $-\theta$ ' $(0)$ ' for positive values of $\theta_{\mathrm{e}}$. (a) $\lambda=0.0, \mathrm{C}=1.0$; (b) $\lambda=0.0, \mathrm{C}=0.5$; (c) $\lambda=0.05, \mathrm{C}=1.0$;

(d) $\lambda=0.05, C=0.5$

\subsection{Other Flow and Heat Transfer Characteristics}

Variations in shear stress ' $f$ " $(\eta)$ 'of the upper solution are presented in Fig. 3(a) and those of the lower solution in the Fig. 3(b). It may be noted that variations in the lower solutions with the parameters are more striking than those in the upper solution. Hydrodynamic boundary layer thickness pertaining to the lower solution can be observed to be much higher than the boundary layer thickness of the upper solution and in fact boundary layer thickness of the lower solution is almost twice that of the upper solution Boundary layer thickness can be observed to be very high when $\theta_{e}=2$, and in the absence of magnetic field. However the maximum value of shear stress of lower solution is smaller than that of the upper solution.

Variations in ' $\theta$ ' $(\eta)$ ' are presented in Fig. 4(a) for the upper solution and in Fig. 4(b) for the lower solution. One important observation is that, for both the solutions, for all values of the parameters, ' $\theta^{\prime}(\eta)$ ' is negative. Thermal boundary layer thickness of the lower solution is much higher than that of the upper solution and in fact, boundary layer thickness of lower solution is almost twice that of the upper solution. It may be observed that absolute value of ' $\theta^{\prime}(\eta)$ ' is maximum when $\theta_{e}=2$ (i.e., for gases). Absolute maximum of the lower solution is smaller than absolute maximum of the upper solution.

Variations in fluid velocity of the upper solution are presented in Fig. 5(a) and of the lower solution in Fig. 5(b). As can be expected, the variations in the shear stress are reflected in the plots of the velocity. However, it may noted that the variations in the lower solution with the parameters are significant than the variations in the upper solution. Hydrodynamic boundary layer thickness of the lower solution is much higher than that of the upper solution.

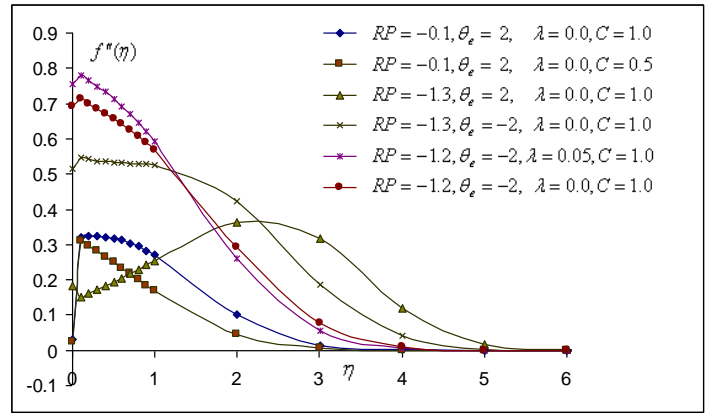

(a)

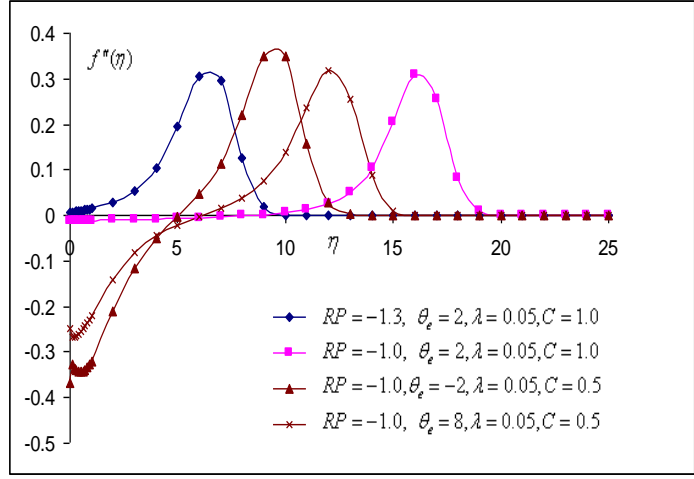

(b)

Fig. 3. Variations in $f^{\prime \prime}(\eta)$. (a) upper solution; (b) lower solution 


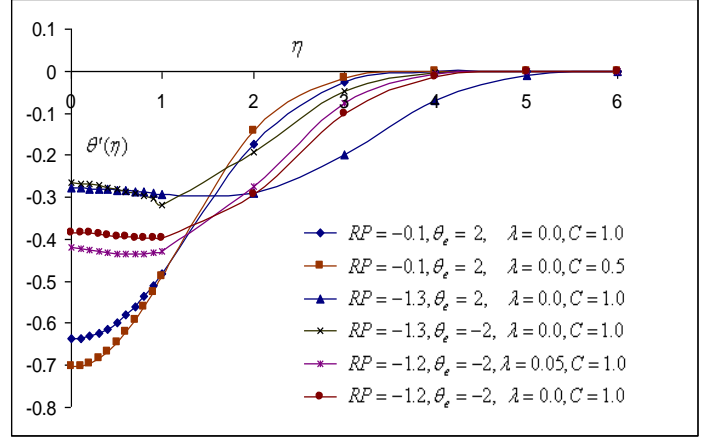

(a)

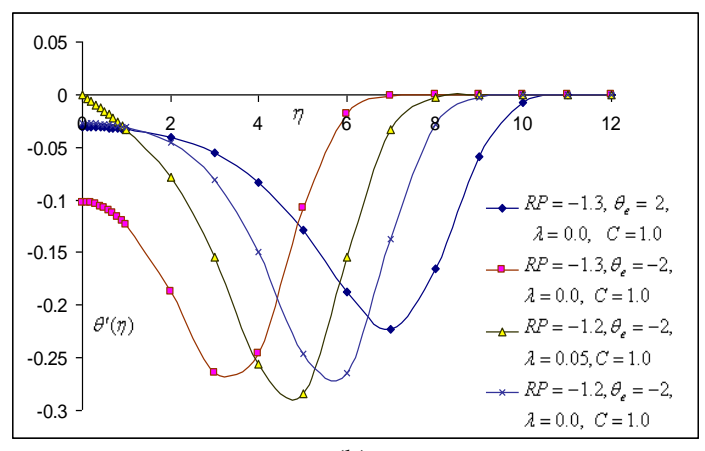

(b)

Fig. 4. Variations in $\theta^{\prime}(\eta)$. (a) upper solution; (b) lower solution

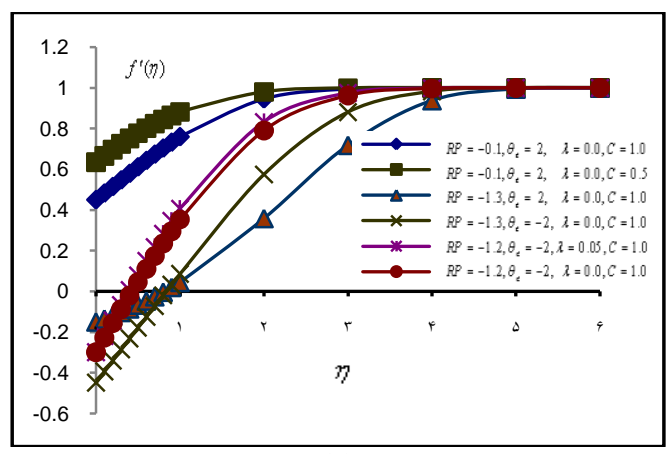

(a)

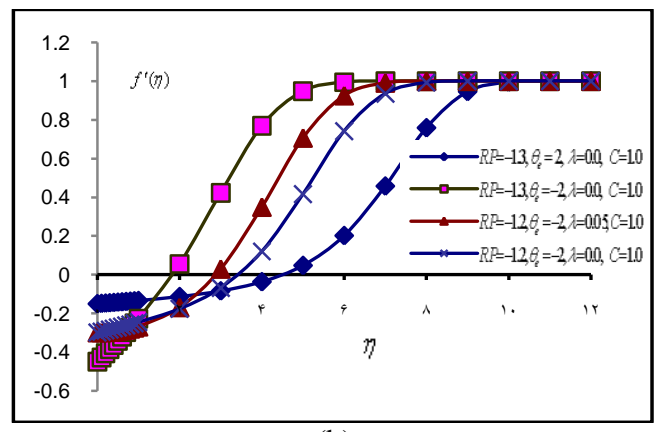

(b)

Fig. 5. Variations in $f^{\prime}(\eta)$. (a) upper solution; (b) lower solution

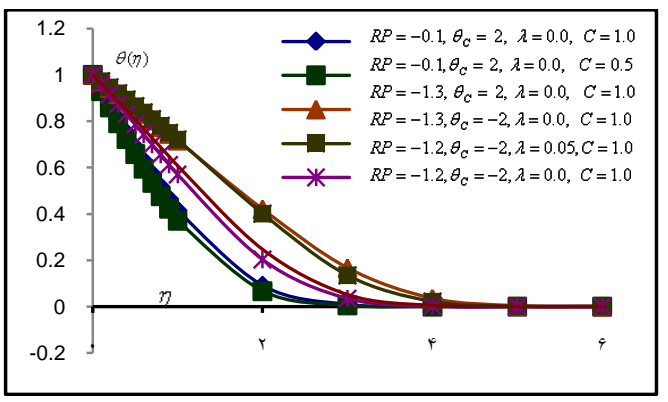

(a)

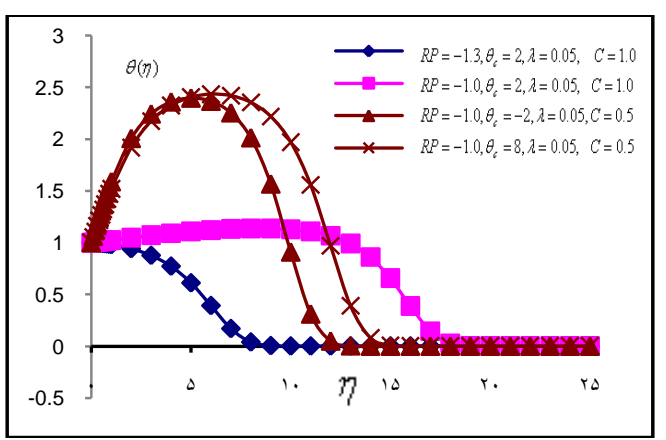

(b)

Fig.6. Variations in $\theta$ ( $)$. (a) upper solution; (b) lower solution

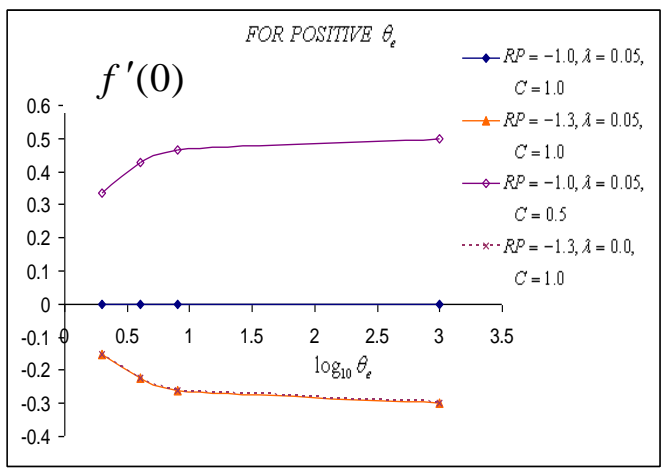

Fig.7. Variations in $f^{\prime}(0)$ for positive values of $\theta_{\mathrm{e}}$

Variations in fluid temperature of the upper and lower solutions are presented in Figs. 6(a) and 6(b) respectively. The variations in the temperature can be anticipated from the variations in ' $\theta^{\prime}(\eta)$ '. Further, variations in the lower solution are more significant than those in the upper solution. Thermal boundary layer thickness of the lower solution is much higher than that of the upper solution.

Variations in slip velocity for positive values of $\theta_{e}$ are presented in Fig. 7. From the figure, it may be noted that the slip velocity can be observed to be more in the presence of magnetic field ( $C=0.5$ ) than in its absence $(C=1)$. For both positive as well as negative values of $\theta_{e}$, in the absence of magnetic field, the slip velocity takes either zero value or negative values while in the presence of magnetic field, slip velocity takes positive values. In the presence of magnetic field, slip velocity increases with increasing positive values of $\theta_{e}$ (i.e., for gases, as $\theta_{e}$ changes from 2 to 1000) while it 
decreases with increasing negative values of $\theta_{e}$ (i.e., for liquids, as $\theta_{e}$ changes from -2 to -8 and further to 1000). However, in the absence of magnetic field, the behavior of slip velocity with $\theta_{e}$ is vice versa.

\subsection{Observations from Tables}

It is noticed that there exists a single solution or dual solution or no solution for different negative values of the mixed convection parameter $R P$. In Table 1 , are presented sets of values of the parameters for which either a single solution or dual solution or no solution exists for the problem. From numerical results as well as from tables, it could be observed that the range of values of $R P$ for which a solution exists is seen to increase with decreasing values of $\theta_{e}$ (either positive or negative). The range can also be seen to increase considerably in the presence of magnetic field $(C=0.5)$ than in its absence $(C=1)$. The range can be seen to increase further in the variable wall temperature case $(\lambda=0.05)$ than in the constant wall temperature case $(\lambda=0)$.

Variations in hydrodynamic boundary layer thickness $\left(\delta_{v}\right)$ and thermal boundary layer thickness $\left(\delta_{t}\right)$ in the absence of magnetic field ( $C=1$ ) are presented in Table 2(a) for $R P=-1.2, \lambda=0.05$. Changes in $\delta_{v}, \delta_{t}$ in the presence of magnetic field $(C=0.5)$ are presented in table 2(b) for $R P=-2.2, \lambda=0.05$.

Table 1 Ranges of values of $R P$. (a) $C=1, \lambda=0.05$ ( no magnetic field, varying wall temperature).

(b) $C=0.5, \lambda=0.05$ ( magnetic field, varying wall temperature)

(a)

\begin{tabular}{|c|c|c|c|c|}
\hline $\begin{array}{l}\text { Parameters } \\
\text { Type } \\
\text { Of solution }\end{array}$ & $\begin{array}{c}\theta_{e}=2 \\
C=1 \\
\lambda=0.05\end{array}$ & $\begin{array}{c}\theta_{e}=8 \\
C=1 \\
\lambda=0.05\end{array}$ & $\begin{array}{c}\theta_{e}=-2 \\
C=1 \\
\lambda=0.05\end{array}$ & $\begin{array}{c}\theta_{e}=-8 \\
C=1 \\
\lambda=0.05\end{array}$ \\
\hline $\begin{array}{l}\text { Single } \\
\text { Solution }\end{array}$ & $-0.7 \leq R P \leq 0$ & $-0.2 \leq R P \leq 0$ & $R P=0$ & $R P=0$ \\
\hline $\begin{array}{l}\text { Dual } \\
\text { Solution }\end{array}$ & $-1.435 \leq R P \leq-0.8$ & $-1.376 \leq R P \leq-0.3$ & $-1.332 \leq R P \leq-0.1$ & $-1.354 \leq R P \leq-0.1$ \\
\hline $\begin{array}{c}\text { No } \\
\text { Solution }\end{array}$ & $R P>-1.435$ & $R P>-1.376$ & $R P>-1.332$ & $R P>-1.354$ \\
\hline
\end{tabular}

(b)

\begin{tabular}{|c|c|c|c|c|}
\hline $\begin{array}{l}\text { Parameters } \\
\text { Type } \\
\text { of solution }\end{array}$ & $\begin{array}{c}\theta_{e}=2 \\
C=0.5 \\
\lambda=0.05\end{array}$ & $\begin{array}{c}\theta_{e}=8 \\
C=0.5 \\
\lambda=0.05\end{array}$ & $\begin{array}{l}\theta_{e}=-2 \\
C=0.5 \\
\lambda=0.05\end{array}$ & $\begin{array}{l}\theta_{e}=-8 \\
C=0.5 \\
\lambda=0.05\end{array}$ \\
\hline $\begin{array}{l}\text { Single } \\
\text { Solution }\end{array}$ & $-1.3 \leq R P \leq 0$ & $-0.3 \leq R P \leq 0$ & $R P=0$ & $R P=0$ \\
\hline $\begin{array}{c}\text { Dual } \\
\text { Solution }\end{array}$ & $-2.81 \leq R P \leq-1.4$ & $-2.74 \leq R P \leq-0.4$ & $-2.6996 \leq R P \leq-0.1$ & $-2.7182 \leq R P \leq-0.1$ \\
\hline $\begin{array}{c}\text { No } \\
\text { Solution }\end{array}$ & $R P>-2.81$ & $R P>-2.74$ & $R P>-2.6996$ & $R P>-2.7182$ \\
\hline
\end{tabular}

Tables 2 Hydrodynamic boundary layer thickness $\left(\delta_{v}\right) \&$ Thermal boundary layer thickness $\left(\delta_{t}\right)$

(a)

\begin{tabular}{|c|c|c|c|c|}
\hline Parameters & $\begin{array}{c}\theta_{e}=2 \quad R P=-1.2 \\
C=1, \lambda=0.05\end{array}$ & $\begin{array}{c}\theta_{e}=8 \quad R P=-1.2 \\
C=1, \lambda=0.05\end{array}$ & $\begin{array}{c}\theta_{e}=-2 \quad R P=-1.2 \\
C=1, \lambda=0.05\end{array}$ & $\begin{array}{cl}\theta_{e}=-8 & R P=-1.2 \\
C=1, & \lambda=0.05\end{array}$ \\
\hline Thickness & \multicolumn{4}{|c|}{ UPPER SOLUTION } \\
\hline$\delta_{v}$ & 8 & 8 & 7 & 8 \\
\hline$\delta_{t}$ & 8 & 8 & 7 & 8 \\
\hline \multicolumn{5}{|c|}{ LOWER SOLUTION } \\
\hline$\delta_{v}$ & 15 & 13 & 11 & 12 \\
\hline$\delta_{t}$ & 15 & 13 & 11 & 12 \\
\hline
\end{tabular}


(b)

\begin{tabular}{|c|c|c|c|c|}
\hline \multirow{2}{*}{ Thickness } & $\begin{array}{c}\theta_{e}=2 \quad R P=-2.2 \\
C=0.5, \lambda=0.05\end{array}$ & $\begin{array}{c}\theta_{e}=8 \\
C=0.5, \lambda=0.05\end{array}$ & $\begin{array}{c}\theta_{e}=-2 \\
C=0.5, \lambda=0.05\end{array}$ & $\begin{array}{c}\theta_{e}=-8 \\
C P=0.5, \lambda=0.05\end{array}$ \\
\cline { 2 - 5 } & \multicolumn{5}{|c|}{ UPPER SOLUTION } \\
\hline$\delta_{v}$ & 8 & 7 & 7 & 7 \\
\hline$\delta_{t}$ & 8 & 7 & 7 & 7 \\
\hline$\delta_{v}$ & 16 & 15 & 14 & 14 \\
\hline$\delta_{t}$ & 16 & 15 & 14 & 14 \\
\hline
\end{tabular}

From the tables, as well as from numerical results, it may be noted that for upper solution, there are negligible changes in $\delta_{v}, \delta_{t}$ with changing values of $\theta_{e} \& \lambda$. However for the lower solution, the changes in $\delta_{v}, \delta_{t}$ are significant with changes in the parameters. Both $\delta_{v}$ and $\delta_{t}$ decrease for positive increasing values and negative decreasing values of $\theta_{e}$. Both $\delta_{v}$ and $\delta_{t}$ assume larger values for larger negative values of $R P$. From numerical results, $\delta_{v}$, $\delta_{t}$ are observed to decrease with increasing negative values of $R P$. Also $\delta_{v}, \delta_{t}$ are observed to assume larger values in the presence of magnetic field than in the absence of magnetic field. These variations of $\delta_{v}$

, $\delta_{t}$ with changing values of $\theta_{e}, \lambda$ and $C$, however, are not the same for all values of $R P$.

\subsection{NM Earlier Works}

Results of the present analysis are in excellent agreement with those of Lai et al. (1990) when $\lambda=0$, $C=1$ for positive values of $R P$ (i.e., in the assisting flow case when the plate temperature is constant and when there is no magnetic field). Results of the present analysis are in excellent agreement with those of Aly et al. (2003) when $C=1, \theta_{e}=1000$ (i.e., when there is no magnetic field and when viscosity is taken to be a constant. It is noticed that 1000 is a sufficiently large value for $\theta_{e}$ that has given results of constant viscosity case). Results of the present analysis also are in excellent agreement with those of Chin et al. (1990) when $C=1$ and $\lambda=0$ (i.e., when the plate temperature is constant and when there is no magnetic field, both in the assisting flow and opposing flow cases).

\section{Conclusion}

In the opposing flow case, there can be a unique solution, dual solutions or no solution for the problem depending on the values of $R P$. In the case of dual solutions, the solution corresponding to a relatively larger value of ' $f^{\prime \prime}(0)$ ' is referred to as the upper solution and the one corresponding to a smaller value of ' $f$ " $(0)$ ' is referred to as the lower solution. The range of values of $R P$ over which solution exists is considerably large in the presence of magnetic field than in its absence. In the isothermal case $(\lambda=0.0)$, ' $f^{\prime \prime}(0)$ ' as well as ' $-\theta^{\prime}(0)$ ' assume only positive values, while in the varying wall temperature case ( $\lambda=0.05$ ), they assume both positive and negative values, indicating that dual solutions exist over a wider range of values of $R P$ in the varying wall temperature case than in isothermal case. Variations in the lower solutions with the parameters are more striking than the variations in the upper solution. Hydrodynamic boundary layer thickness pertaining to the lower solution is much higher than the boundary layer thickness of the upper solution. Thermal boundary layer thickness of the lower solution is also much higher than that of the upper solution. Drag is less in the presence of magnetic field than its absence. Drag is less in the isothermal case than in the varying wall temperature case. Heat transfer coefficient assumes larger values in the presence of magnetic field than in its absence. The range of values of $R P$ for which a solution exists increases with decreasing values of $\theta_{e}$ (either positive or negative).

\section{ACKNOWLEDGEMENTS}

The authors wish to convey their thanks to the Management and Principal of S.R.K.R. Engineering College, Bhimavaram for providing the facilities.

\section{REFERENCES}

Acharya, M., G.C. Dash and L.P. Singh., Magnetic Field Effects on the Free Convection and Mass Transfer Flow through Porous Medium with Constant Suction and Constant Heat Flux, Indian J. Pure Appl. Math., 31(2000) 1-18.

Aly, E.H., L. Elliot and D.B. Ingham., Mixed convection boundary layer Flow over a vertical surface embedded in a porous medium, European Journal of Mechanics B/Fluids., 22(2003) $529-543$.

Carey, V.P., and J.C. Mollendorf., Variable Viscosity Effects in several natural convection flows, Int.J.Heat-Mass Transfer, 23(1980) 95 -109.

Chen, C.H., and C.K. Chen., Non-Darcian mixed convection along a vertical plate embedded in a porous medium. Appl. Math. Modelling 14(1990) 482- 488. 
Cheng, P., and I.Dee chang., Buoyancy Induced Flows in a Porous Medium Adjacent to Impermeable Horizontal surfaces, Hawaii Geothermal Project technical report no. 12 (1975).

Cheng, P., and W.J. Minkowycz., Free Convection about a Vertical Flat Plate Embedded in a porous medium with app.lication to heat transfer from a Dike, J.Geophys. Res.82 (1977), 2040 2044.

Chin, K.E., R. Nazar., N.M. Arifin and I. Pop., Effect of variable viscosity on mixed convection boundary layer flow over a vertical surface embedded in a porous medium, International Communications in Heat and Mass transfer., 34(2007) $464-473$.

Rao, C.N.B., V. Laksmi Prasannam and T. Rajarani., Mixed Convection in a Porous Medium with Magnetic Field, Variable Viscosity and Varying wall Temperature. International Journal of Computational Mathematical Ideas, 2(1) (2010)13-21.

Joshi, Y., and B. Gebhart., Mixed convection in porous media adjacent to a vertical uniform heat flux surface. Int.J. Heat Mass Transfer 28(1985) 1783-1786.

Lai, F.C., and F.A. Kulacki., The effect of Variable Viscosity on Convective Heat Transfer Along a Vertical Surface in a Saturated Porous Medium, Int. J. Heat Mass Transfer. 33(1990)1028 1031.

Merkin, J.H., Mixed Convection Boundary Layer Flow on a vertical surface in a saturated porous medium, Journal of Engineering Mathematics, 14(1980) 301-313.

Minkowycz, W.J., and P. Cheng., Free Convection about a Vertical Cylinder embedded in a Porous Medium, Int.J.Heat Mass Transfer, 19 (1976) $805-813$.

Pu, W.L., P. Cheng and T.S. Zhao., An Experimental Study of Mixed Convection Heat Transfer in Vertical Packed Channels, AIAA Journal of Thermo Physics and Heat Transfer, 13 (4)(1999) 517-521.

Ranganathan, R., and R. Viskanta., Mixed convection boundary- layer flow along a vertical surface in a porous medium. Numer. Heat Transfer 7(1984) 305- 317.

Sobha, V.V., and K. Ramakrishna., Convective Heat Transfer Past a Vertical Plate embedded in a Porous Medium with an Applied Magnetic Field, IE(I) Journal - MC, 84(2003) 130 - 134. 